\title{
Histologic Analysis of Retrieved Clots in Acute Ischemic Stroke: Correlation with Stroke Etiology and Gradient-Echo MRI
}

\author{
S.K. Kim, DW. Yoon, T.S. Kim, H.S. Kim, T.W. Heo, and M.S. Park
}

\begin{abstract}
BACKGROUND AND PURPOSE: It is unclear whether clot composition analysis is helpful to predict a stroke mechanism in acute large vessel occlusion. In addition, the relationship between early vessel signs on imaging studies and clot compositions has been poorly understood. The purpose of this study was to elucidate the relationship between clot composition and stroke etiology following mechanical thrombectomy and to investigate the effect of varied clot compositions on gradient-echo MR imaging of clots.
\end{abstract}

MATERIALS AND METHODS: Histopathologic analysis of retrieved clots from 37 patients with acute MCA occlusion was performed. Patients underwent gradient-echo imaging before endovascular therapy. Retrieved clots underwent semiquantitative proportion analysis to quantify red blood cells, fibrin, platelets, and white blood cells by area. Correlations between clot compositions and stroke subtypes and susceptibility vessel signs on gradient-echo imaging were assessed.

RESULTS: Stroke etiology was classified as cardioembolism in 22 patients (59.4\%), large-artery atherosclerosis in 8 (21.6\%), and undetermined in 7 (18.9\%). The clots from cardioembolism had a significantly higher proportion of red blood cells $(37.8 \%$ versus $16.9 \%, P=.031)$ and a lower proportion of fibrin $(32.3 \%$ versus $48.5 \%, P=.044)$ compared with those from large-artery atherosclerosis. The proportion of red blood cells was significantly higher in clots with a susceptibility vessel sign than in those without it (48.0\% versus $1.9 \%, P<.001)$, whereas the proportions of fibrin $(26.4 \%$ versus $57.0 \%, P<.001)$ and platelets $(22.6 \%$ versus $36.9 \%, P=.011)$ were significantly higher in clots without a susceptibility vessel sign than those with it.

CONCLUSIONS: The histologic composition of clots retrieved from cerebral arteries in patients with acute stroke differs between those with cardioembolism and large-artery atherosclerosis. In addition, a susceptibility vessel sign on gradient-echo imaging is strongly associated with a high proportion of red blood cells and a low proportion of fibrin and platelets in retrieved clots.

ABBREVIATIONS: GRE $=$ gradient-echo; $\mathrm{RBC}=$ red blood cell; $\mathrm{WBC}=$ white blood cell

$E^{n}$ ndovascular therapy is increasingly used for treating acute ischemic stroke due to intracranial large-vessel occlusion. Among various endovascular therapies, mechanical thrombectomy is now accepted as the first-line endovascular therapy for acute large-vessel occlusion. Recent studies have shown that thrombectomies with a retrievable stent or flexible aspiration catheter were associated with high-recanalization and low-complication rates. ${ }^{1-5}$ One of the striking features of mechanical

Received December 19, 2014; accepted after revision February 6, 2015. From the Departments of Radiology (S.K.K., W.Y., T.W.H.), Neurosurgery (T.S.K.), Forensic Medicine (H.S.K.), and Neurology (M.S.P.), Chonnam National University Medical School, Chonnam National University Hospital, Gwangju, Republic of Korea.

Please address correspondence to Woong Yoon, MD, Department of Radiology, Chonnam National University Hospital, 671 Jebong-Ro, Dong-Gu, Gwangju, 501-757, Republic of Korea; e-mail: radyoon@jnu.ac.kr

http://dx.doi.org/10.3174/ajnr.A4402 thrombectomy is that it enables physicians to perform histopathologic examination of clots retrieved from human intracranial arteries. Recent studies suggested that histologic examination of retrieved clots can offer new insights into the pathogenesis of acute stroke due to intracranial large-vessel occlusion..$^{6-9}$ However, it is currently unclear whether clot-composition analysis is helpful to predict a stroke mechanism in acute large-vessel occlusion. Only a few studies have been conducted on this topic, and prior studies have yielded only vague and contradictory results. ${ }^{8-10}$

In addition, histopathologic analysis of retrieved clots allows understanding of the pathologic basis of early vessel signs on imaging studies in patients with acute ischemic stroke. Several studies have shown that the hyperattenuated vessel sign on CT and the susceptibility vessel sign on gradient-echo (GRE) MR imaging were more often associated with erythrocyte-rich thrombi (red thrombi) than fibrin-rich thrombi (white thrombi). ${ }^{8,10,11}$ Platelets play an important role in the pathogenesis of white thrombi 
associated with atherosclerotic plaque rupture. ${ }^{12}$ However, the relationship between early vessel signs on imaging studies and the proportion of platelets within the retrieved clots has been poorly understood. Thus, we performed histopathologic analysis on retrieved clots from patients with acute MCA occlusion to further elucidate the relationship between clot composition and stroke etiology and to investigate the effect of platelet levels on GRE MR imaging of clots.

\section{MATERIALS AND METHODS \\ Patients}

From January 2011 to October 2012, 56 consecutive patients presenting with acute stroke due to MCA occlusions were treated with mechanical thrombectomy, including stent-based thrombectomy and manual aspiration thrombectomy at a tertiary university hospital. Patients underwent a nonenhanced CT scan and multimodal MR imaging before endovascular thrombectomy procedures. Of these patients, those who had GRE images of insufficient quality for reliable evaluation $(n=7)$ or did not have thrombus material suitable for histopathologic analyses $(n=12)$ were excluded. This step left 37 patients in this study. We prospectively collected the following clinical and radiologic data of these 37 patients: demographic features, cerebrovascular risk factors, NIHSS scores on admission and at discharge, use of IV thrombolysis, time to endovascular treatment, procedure time, time to reperfusion, revascularization status, clinical outcomes, and stroke subtypes.

Subtypes of ischemic stroke were determined at the time of discharge by stroke neurologists according to the classification of the Trial of Org 10172 in Acute Stroke Treatment. ${ }^{13}$ Large-artery atherosclerosis was defined as "catheter angiographic" findings showing $>50 \%$ stenosis or occlusion of the ipsilateral extracranial or intracranial carotid artery proximal to the occlusion site without evidence of potential sources of cardioembolism in other diagnostic studies. Moderate or severe ( $>50 \%$ ) underlying intracranial atherosclerotic stenosis was also regarded as large-artery atherosclerosis. Cardioembolsim was defined as having at least 1 of the following predisposing factors: atrial fibrillation or flutter, left atrial thrombus, a prosthetic valve, severe mitral stenosis, a patent foramen ovale, concomitant acute myocardial infarction, congestive heart failure, infective endocarditis, and sick sinus syndrome, in the absence of moderate or severe (>50\%) ipsilateral arterial stenosis on imaging studies. The institutional ethics committee approved this study. For each patient, written informed consent for endovascular therapy and histopathologic analysis of retrieved thrombi was obtained from a family member.

\section{Endovascular Treatment}

On admission, a stroke neurologist performed a neurologic assessment based on the NIHSS. The inclusion criteria for endovascular thrombectomy were as follows: presentation within 6 hours of stroke onset; baseline NIHSS score of $\geq 4$; no intracranial hemorrhage detected on cranial CT or MR imaging; a target mismatch pattern on multimodal MR imaging based on visual estimation (time-to-peak map of perfusion imaging showing a lesion volume $\geq 30 \%$ larger than that detected with diffusion imaging); and infarct volume on diffusion imaging or nonenhanced CT of less than one-third of the MCA territory.
Cerebral angiography and endovascular therapy were performed with the patient under conscious sedation. In cases of agitation, an intravenous bolus of midazolam was given and repeated if necessary. Stent-based thrombectomy with a Solitaire stent (Covidien, Irvine, California) was performed as the first-line endovascular treatment. When stent-based thrombectomy was unsuccessful, additional mechanical approaches were performed, including manual aspiration thrombectomy with a Penumbra reperfusion catheter (Penumbra, Alameda, California). The details of the techniques used for stent-based thrombectomy and manual aspiration thrombectomy were previously described. ${ }^{5,14}$ Revascularization status was assessed on the final angiogram and was classified according to the modified TICI scale, ${ }^{15}$ and successful revascularization was defined as a modified TICI grade of $2 \mathrm{~b}$ or 3. Clinical outcome was assessed by a stroke neurologist by using the mRS during an outpatient visit 3 months after treatment. If patients were unable to attend the outpatient clinic, outcomes were obtained via telephone interview. A good clinical outcome was defined as an mRS score of $\leq 2$.

\section{MR Imaging Analysis}

MR imaging examinations were performed by using a $1.5 \mathrm{~T}$ unit (Signa HDxt; GE Healthcare, Milwaukee, Wisconsin). Before the endovascular procedure, patients underwent MR imaging including DWI, GRE, FLAIR, 3D TOF MRA, and perfusion imaging. GRE sequence parameters included a TR of $750-800 \mathrm{~ms}$, TE of 14 $\mathrm{ms}$, flip angle of $20^{\circ}$, section thickness of $4 \mathrm{~mm}$, intersection gap of $0 \mathrm{~mm}$, and FOV of $230 \times 230 \mathrm{~mm}$. All GRE images were retrospectively reviewed by 2 neuroradiologists (with 2 and 4 years of experience, respectively) who were blinded to the findings of conventional angiography. Conclusions were reached by consensus regarding the presence or absence of the susceptibility vessel sign on GRE imaging. A positive susceptibility vessel sign was defined as a hypointense signal change on GRE imaging within the occluded MCA, in which the diameter of the hypointense signals exceeded that of the contralateral MCA. ${ }^{16,17}$ A negative susceptibility vessel sign was defined as an absence of a positive susceptibility vessel sign in the occluded MCA.

\section{Histopathologic Analysis}

Retrieved clot material was fixed in 10\% neutralized buffered formalin immediately after retrieval. Formalin-fixed, paraffin-embedded tissue was cut at $4-\mu \mathrm{m}$ thickness. All clots were stained with hematoxylin-eosin. Then, deparaffinized sections were immunohistochemically stained with antibodies for platelet glycoprotein IIIa, CD61 (LifeSpan Biosciences, Seattle, Washington). Immunostains were performed by the routine avidin-biotin complex method. Immunoreactivity was visualized with a diaminobenzidine (DAB; Dako, Glostrup, Denmark) solution and then counterstained with hematoxylin.

Stained slides were scanned at $\times 200$ magnification by using a Scanscope XT digital scanner (Apergio, Vista, California). ImageJ software (National Institutes of Health, Bethesda, Maryland) was used for semiquantitative analysis of the percentage of red blood cells (RBCs), fibrin, platelets, and white blood cells (WBCs) by area. Histopathologic analysis was performed by an experienced 

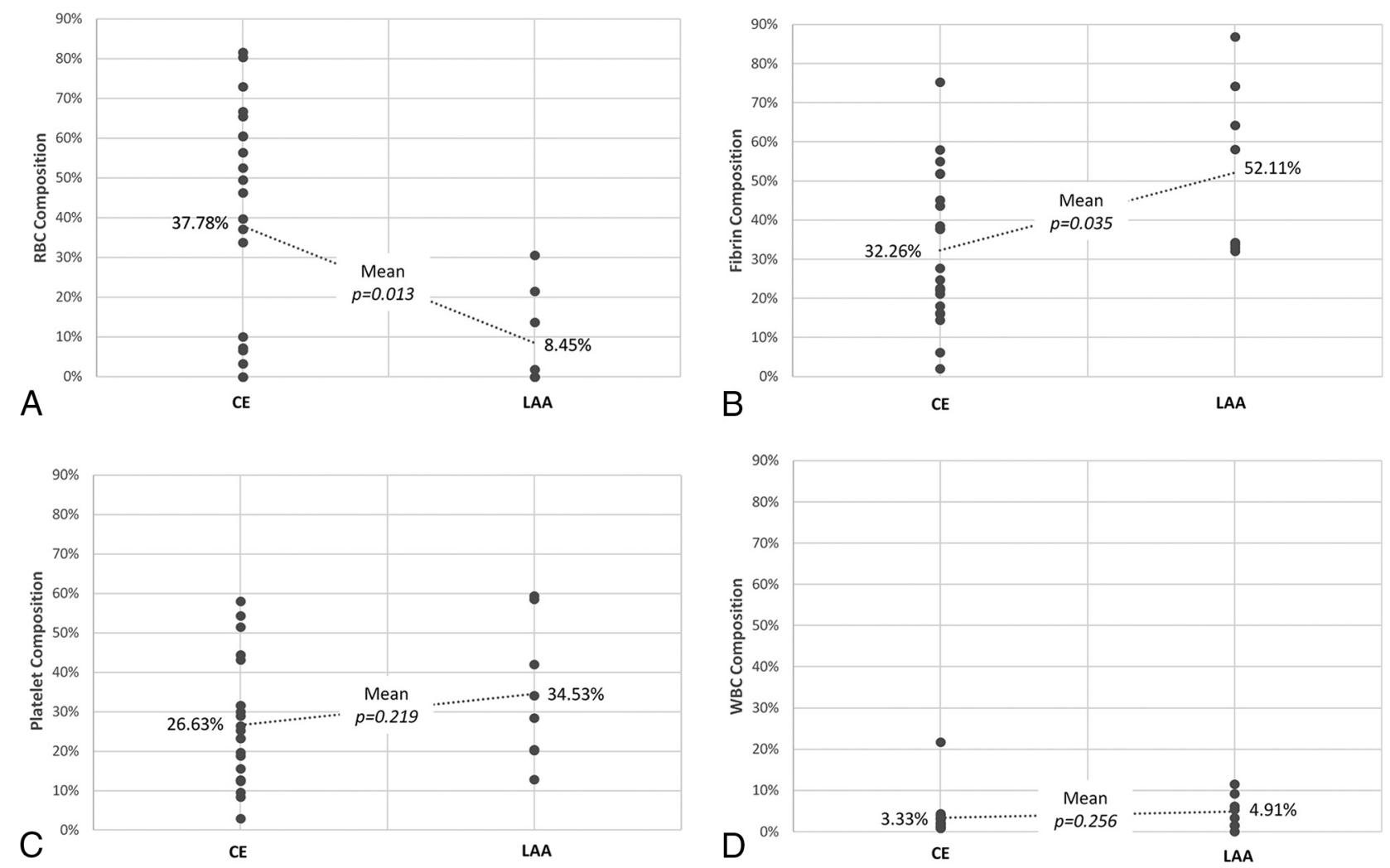

FIG 1. Differences in the mean percentages of clot compositions between cardioembolism (CE) and large-artery atherosclerosis (LAA). Red blood cell $(A)$, fibrin $(B)$, platelet $(C)$, and white blood cell $(D)$ composition.

pathologist without knowledge of the patient characteristics and imaging findings.

\section{Statistical Analysis}

Continuous data are presented as means or medians with SDs, and categoric data are presented as frequencies and percentages. Percentages of each specific clot component were regarded as continuous variables. First, the percentages of each clot component were compared between patients with cardioembolism and those with large-artery atherosclerosis. Then, the percentages of RBCs, fibrin, platelets, and WBCs were compared between patients with a positive susceptibility vessel sign on GRE imaging and those with a negative susceptibility vessel sign. The $\chi^{2}$ or Fisher exact test was used for categoric variables, and the MannWhitney $U$ or Kruskal-Wallis test was used for continuous variables. All statistical analyses were performed with SPSS software (Version 21.0; IBM, Armonk, New York). $P<.05$ indicated a statistically significant difference.

\section{RESULTS}

During the study period, 37 eligible patients (20 men and 17 women; mean age, 69 years; age range, 40-91 years) were identified. In our study group, 68\% (25/37) of patients had hypertension, 51\% (19/37) had atrial fibrillation, 32\% (12/37) had dyslipidemia, 24\% (9/37) had a history of ischemic stroke, 16\% (6/37) had a smoking history, 11\% (4/37) had diabetes mellitus, 8\% (3/ 37 ) had a history of coronary artery disease, and 5\% (2/37) had congestive heart failure.

The median NIHSS score on admission was 15 , with scores ranging from 5 to 19 . The median time from symptom onset to endovascular therapy was 240 minutes (range, 60-360 minutes), the median procedure time was 30 minutes (range, 10-90 minutes), and the median time to reperfusion was 257 minutes (range, 93-420 minutes). IV rtPA was administered in 23 patients $(62 \%)$ before mechanical thrombectomy. Of 37 patients, 5 had a tandem occlusion at the proximal cervical portion of the ICA and received carotid artery stent placement before the intracranial revascularization procedure. Successful reperfusion (modified TICI $2 \mathrm{~b}$ or 3$)$ was achieved in $83.7 \%$ (31/37) of patients, and complete reperfusion (modified TICI grade 3 ) occurred in $70.2 \%(26 / 37)$ of patients. No patient had symptomatic hemorrhage during the hospital stay. At discharge, the NIHSS score improved (decrease of $\geq 4$ points) in 28 patients ( $75.6 \%)$. The median NIHSS score at discharge was 5 . At the 3 -month follow-up, 16 patients (43.2\%) showed a good clinical outcome (mRS 0-2). Mortality was $14 \%$ $(5 / 37)$ at 3 months.

On histopathologic examination, the mean percentage of components across all retrieved clots was $29.3 \pm 29.33 \%$ RBCs, $38.8 \pm 22.64 \%$ fibrin, $28.4 \pm 14.89 \%$ platelets, and $3.5 \pm 3.90 \%$ WBCs. Cholesterol crystals or calcified material was not identified in extracted clots in this study.

The subtypes of stroke were classified as cardioembolism in 22 (59.4\%), large-artery atherosclerosis in $8(21.6 \%)$, and undetermined in 7 (18.9\%). The percentages of RBC and fibrin composition differed significantly between patients with cardioembolism and those with large-artery atherosclerosis (Fig 1). The clots in patients with a cardioembolism had a higher proportion of 

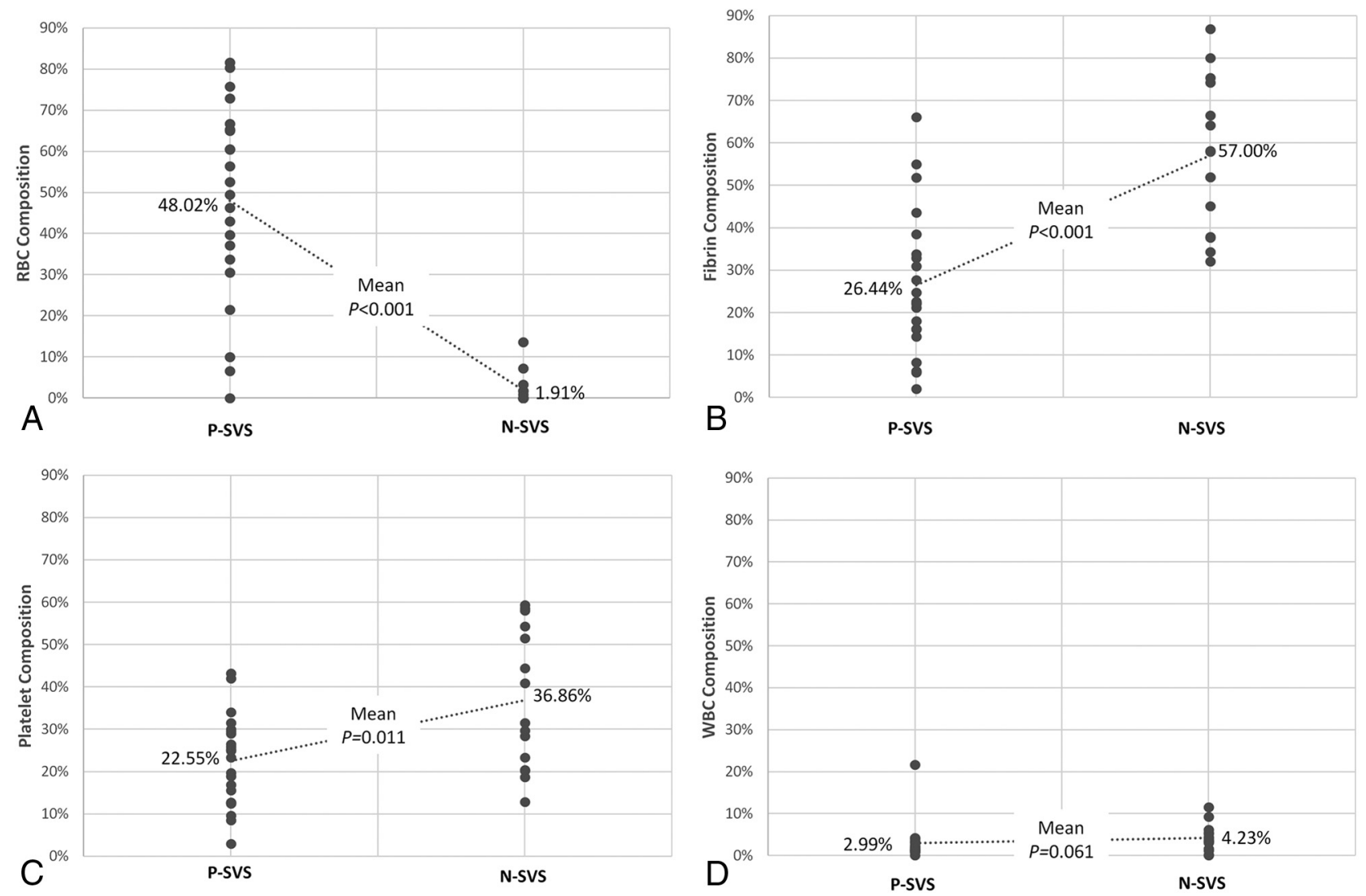

FIG 2. Differences in the mean percentages of clot compositions relative to a positive susceptibility vessel sign (P-SVS) and a negative susceptibility vessel sign (N-SVS). Red blood cell (A), fibrin (B), platelet (C), and white blood cell (D) composition.

RBCs $(37.8 \%$ versus $8.5 \%, P=.013)$ and a lower proportion of fibrin $(32.3 \%$ versus $52.1 \%, P=.035)$ than in those with largeartery atherosclerosis. There were no significant differences in the proportion of platelets $(26.6 \%$ versus $34.5 \%)$ and WBCs $(3.3 \%$ versus $4.9 \%$ ) between patients with a cardioembolism and those with large-artery atherosclerosis. There were no correlations between the proportion of clot components and initial stroke severity or subsequent revascularization status or clinical outcome. The mean percentage of clot components in patients with undetermined etiology was $26.6 \pm 33.81 \%$ RBCs, $44.3 \pm 29.59 \%$ fibrin, $26.7 \pm 7.99 \%$ platelets, and $2.4 \% \pm 2.07 \%$ WBCs. There were no significant differences in the proportion of clot components between patients with an undetermined etiology and those with a cardioembolism or large-artery atherosclerosis.

At GRE imaging, a positive susceptibility vessel sign was identified in 22 (59.4\%) of 37 patients. The relationships between clot component proportion and susceptibility vessel sign on GRE imaging are shown in Fig 2. The mean percentage of RBC composition was higher in clots with a positive susceptibility vessel sign (48.0\% versus $1.9 \%, P<.001$ ) (Fig 3 ), whereas the percentages of fibrin composition $(26.4 \%$ versus $57.0 \%, P<.001)$ and platelets (22.6\% versus $36.9 \%, P=.011$ ) were significantly higher in clots with a negative susceptibility vessel sign (Fig 4). The percentage of WBC composition was consistently low in all clots and was not statistically different between clots with a positive susceptibility vessel sign and those with a negative susceptibility vessel sign (3.0\% versus $4.2 \%$ ).

\section{DISCUSSION}

This study demonstrated that histologic compositions of clots from patients with acute stroke differ between 2 major subtypes of ischemic stroke, namely, large-artery atherosclerosis and cardioembolism. To date, very few studies investigating a possible relationship between the histology of retrieved clots and stroke mechanisms have been reported. ${ }^{8-10}$ Moreover, the results of previous studies have been inconsistent and confusing. Liebeskind et $\mathrm{al}^{10}$ performed histopathologic analysis of retrieved clots in $50 \mathrm{pa}-$ tients with acute ischemic stroke who had undergone endovascular thrombectomy. They briefly stated that clot composition was unrelated to the final determination of stroke etiology; however, they did not provide detailed data on this issue. More recently, Niesten et $\mathrm{al}^{8}$ investigated 22 thrombi retrieved after mechanical thrombectomy in patients with acute stroke. In contrast to our study, they reported that clots originating from large-artery atherosclerosis had the highest percentage of RBCs compared with other stroke subtypes, while there were no significant differences in the proportion of fibrin and platelets between different stroke subtypes. In their study, the patient population included a small number of patients with large-artery atherosclerosis $(n=8)$ and cardioembolism $(n=6)$, which was the major limitation. The most recent study by Boeckh-Behrens et $\mathrm{al}^{9}$ investigated the histopathology of retrieved clots from 34 patients with acute anterior circulation stroke. They reported that there was a significantly higher proportion of WBCs in the thrombi of patients with cardioembolism compared with those with other stroke subtypes. In 

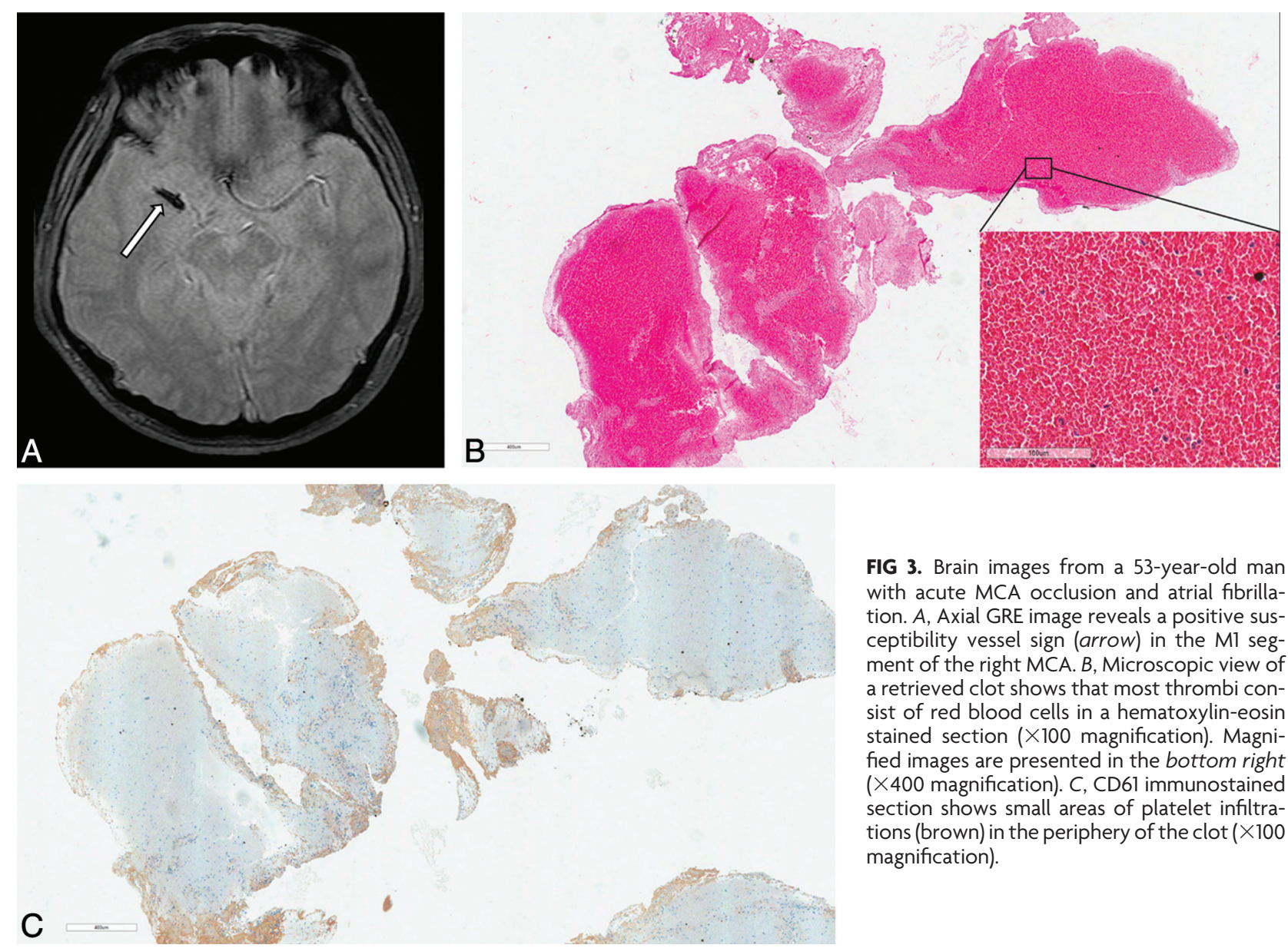

FIG 3. Brain images from a 53-year-old man with acute MCA occlusion and atrial fibrillation. A, Axial GRE image reveals a positive susceptibility vessel sign (arrow) in the M1 segment of the right MCA. $B$, Microscopic view of a retrieved clot shows that most thrombi consist of red blood cells in a hematoxylin-eosin stained section ( $\times 100$ magnification). Magnified images are presented in the bottom right ( $\times 400$ magnification). C, CD61 immunostained section shows small areas of platelet infiltrations (brown) in the periphery of the clot $(\times 100$ magnification).

their study, the quantitative composition of all thrombi consisted of $60 \%$ fibrin, $32 \% \mathrm{RBC}$, and $8 \% \mathrm{WBC}$, and the patient population included 16 patients with cardioembolism and only 3 with large-artery atherosclerosis. They did not provide detailed data regarding proportions of RBCs and fibrin between different stroke subtypes.

In contrast to those previous studies, our study supports the traditional concept that cardioembolic thrombi forming in regions of stasis or slow flow are mainly composed of entrapped RBCs, and thrombi occurring in the context of atherosclerotic large arteries are mainly composed of fibrin and platelets, ${ }^{12,18}$ in the setting of acute ischemic stroke. In our study, we found that the proportion of RBC composition in retrieved clots was higher in patients with cardioembolism compared with those with largeartery atherosclerosis, whereas the proportion of fibrin composition was higher in patients with large-artery atherosclerosis compared with those with cardioembolism. There were no differences in proportions of platelets and WBCs between large-artery atherosclerosis and cardioembolism in our study. In the coronary circulation, most of the thrombi detected at atherosclerotic plaque rupture sites consisted of a mixture of fibrin and platelets, and fibrin was more abundant. ${ }^{19}$ The results from our study are exactly in accordance with those in a previous postmortem study by Sato et al, ${ }^{18}$ which examined the cerebral arteries and thrombi of 17 patients who had died of cardioembolic $(n=11)$ and largeartery atherosclerotic $(n=6)$ strokes within 30 days of stroke onset. They found that the ratio and total area of RBCs in thrombi were significantly larger in cardioembolic than in large-artery atherosclerotic stroke and that the ratio of the area of fibrin was 3 -fold larger in large-artery atherosclerotic than in cardioembolic stroke.

Our study also confirmed the relationship between clot composition and early vessel signs on MR imaging. Paramagnetic intracellular deoxyhemoglobin in acute clots leads to a nonuniform magnetic field and resultant rapid dephasing of spins, which causes marked signal loss on GRE imaging - that is, a susceptibility vessel sign. ${ }^{16,20}$ Only 1 previous study investigated the relationship between retrieved clot composition and susceptibility vessel signs on GRE imaging. Liebeskind et $\mathrm{al}^{10}$ correlated susceptibility vessel signs on GRE imaging and the composition of retrieved clots in 32 patients with acute MCA stroke. In their study, a susceptibility vessel sign on GRE imaging occurred in 56\% (17/ 32) of patients. They found that a susceptibility vessel sign was more common in RBC-dominant and mixed clots compared with fibrin-dominant clots and the mean percentage of RBCs was greater in clots with a susceptibility vessel sign than in those without a susceptibility vessel sign. These results are in line with those in the present study. In our study, a susceptibility vessel sign occurred in 59\% (22/37) of patients. The mean percentage of RBC component was significantly higher in clots with a positive susceptibility vessel sign than in those with a negative susceptibility vessel sign, whereas the percentage of fibrin component was significantly higher in clots with a negative susceptibility vessel sign than in those with a positive susceptibility vessel sign. Moreover, 

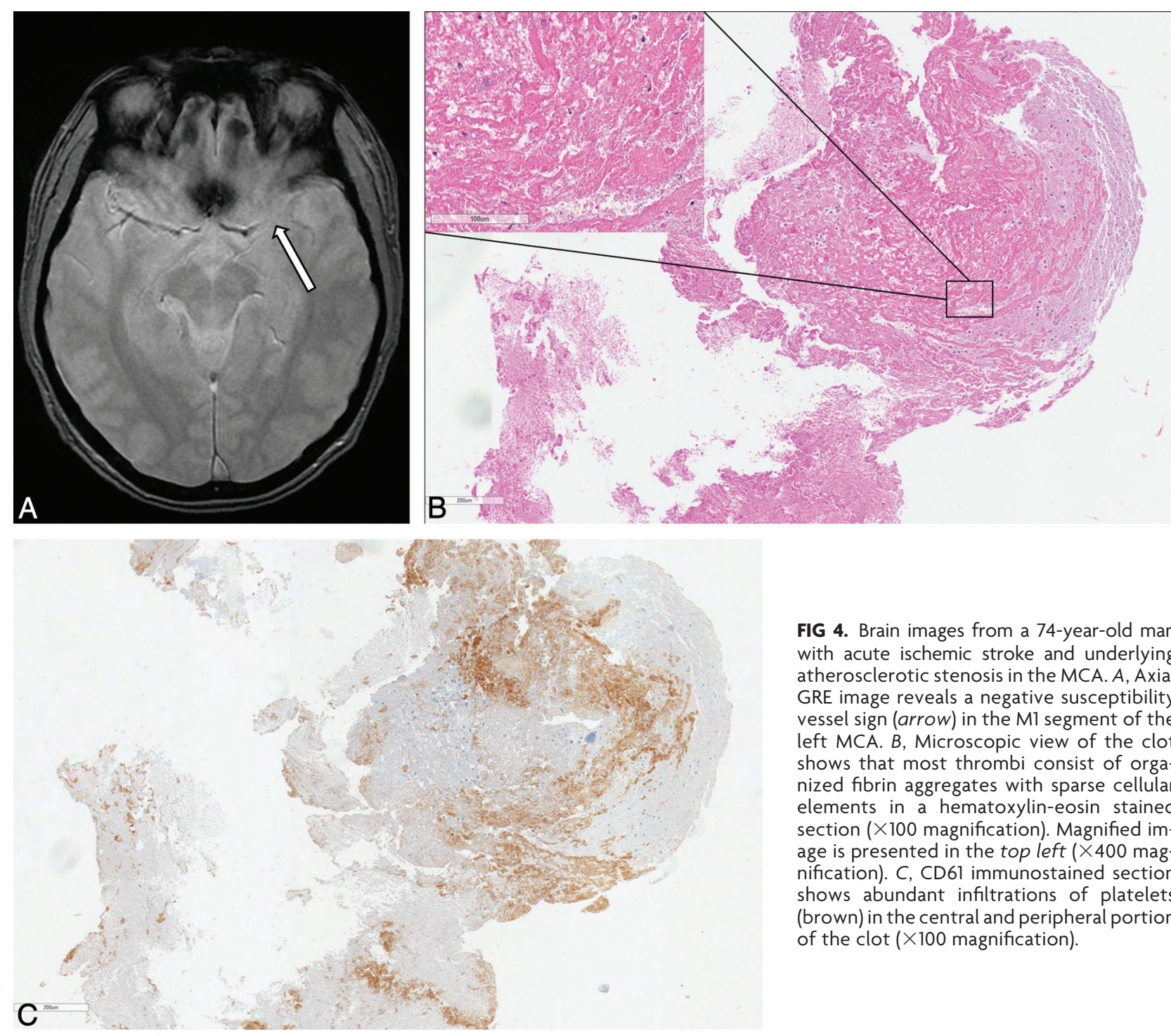

FIG 4. Brain images from a 74-year-old man with acute ischemic stroke and underlying atherosclerotic stenosis in the MCA. A, Axial GRE image reveals a negative susceptibility vessel sign (arrow) in the Ml segment of the left MCA. B, Microscopic view of the clot shows that most thrombi consist of organized fibrin aggregates with sparse cellular elements in a hematoxylin-eosin stained section ( $\times 100$ magnification). Magnified image is presented in the top left ( $\times 400$ magnification). C, CD61 immunostained section shows abundant infiltrations of platelets (brown) in the central and peripheral portion of the clot ( $\times 100$ magnification).

we found that the proportion of platelets was significantly different between clots with and without a susceptibility vessel sign on GRE imaging. In our study, the proportion of platelets was significantly higher in clots with a negative susceptibility vessel sign than in those with a positive susceptibility vessel sign; this finding has not been reported previously.

Platelets were not investigated in the study of Liebeskind et al. ${ }^{10}$ Only 1 study investigated the platelet component in retrieved clots with an immunohistochemical study. Niesten et $\mathrm{al}^{8}$ performed CD31 immunostaining to investigate platelet composition in retrieved clots, in addition to hematoxylin-eosin staining, and correlated clot compositions with attenuation on noncontrast CT. They found a nonsignificant, weak negative correlation between CT attenuation and the proportion of platelets.

Our study has several limitations. The size of the study population was too small to draw a strong conclusion. The number of patients with large-artery atherosclerosis was relatively small compared with those with cardioembolism. During the study period, many cases were excluded because of a lack of retrieved thrombi or insufficient thrombi or incomplete imaging. In addition, successful reperfusion occurred in $84 \%$ of patients in the present study. Thus, we could not evaluate whole clots occluding the MCA in $16 \%$ of cases. Finally, clot composition might be affected by medical treatment in patients before the stroke onset and intravenous rtPA given before mechanical thrombectomy. ${ }^{10}$

\section{CONCLUSIONS}

This study suggests that the histologic composition of clots retrieved from cerebral arteries in patients with acute stroke is different between stroke subtypes. We found that the proportion of RBC composition in retrieved clots is higher in patients with cardioembolism compared with those with large-artery atherosclerosis, whereas the proportion of fibrin composition is higher in patients with largeartery atherosclerosis compared with those with cardioembolism. In addition, a susceptibility vessel sign on GRE MR imaging is strongly associated with a high proportion of RBCs and a low proportion of fibrin and platelets in retrieved clots. These findings would be helpful for clinicians in predicting clot composition and stroke etiology with GRE imaging before endovascular therapy in patients with acute ischemic stroke. 


\section{REFERENCES}

1. Saver JL, Jahan R, Levy EI, et al; SWIFT Trialists. Solitaire flow restoration device versus the Merci retriever in patients with acute ischaemic stroke (SWIFT): a randomised, parallel-group, non-inferiority trial. Lancet 2012;380:1241-49

2. Nogueira RG, Lutsep HL, Gupta R, et al; TREVO 2 Trialists. Trevo versus Merci retrievers for thrombectomy revascularisation of large vessel occlusions in acute ischaemic stroke (TREVO 2): a randomised trial. Lancet 2012;380:1231-40

3. Pereira VM, Gralla J, Davalos A, et al. Prospective, multicenter, single-arm study of mechanical thrombectomy using Solitaire Flow Restoration in acute ischemic stroke. Stroke 2013;44:2802-07

4. Turk AS, Frei D, Fiorella D, et al. ADAPT FAST study: a direct aspiration first pass technique for acute stroke thrombectomy. $\mathrm{J} \mathrm{Neu-}$ rointerv Surg 2014;6:260-64

5. Kim SK, Yoon W, Moon SM, et al. Outcomes of manual aspiration thrombectomy for acute ischemic stroke refractory to stent-based thrombectomy. J Neurointerv Surg 2015;7:473-77

6. Marder VJ, Chute DJ, Starkman S, et al. Analysis of thrombi retrieved from cerebral arteries of patients with acute ischemic stroke. Stroke 2006;37:2086-93

7. Mehta BP, Nogueira RG. Should clot composition affect choice of endovascular therapy? Neurology 2012;79(suppl 1):S63-67

8. Niesten JM, van der Schaaf IC, van Dam L, et al. Histopathologic composition of cerebral thrombi of acute stroke patients is correlated with stroke subtype and thrombus attenuation. PLoS One 2014;9:e88882

9. Boeckh-Behrens T, Schubert M, Förschler A, et al. The impact of histological clot composition in embolic stroke. Clin Neuroradiol 2014 Sep 27. [Epub ahead of print]

10. Liebeskind DS, Sanossian N, Yong WH, et al. CT and MRI early vessel signs reflect clot composition in acute stroke. Stroke 2011;42: 1237-43
11. Simons N, Mitchell P, Dowling R, et al. Thrombus composition in acute ischemic stroke: a histopathological study of thrombus extracted by endovascular retrieval. J Neuroradiol 2015;42:86-92

12. Viereck J, Ruberg FL, Qiao Y, et al. MRI of atherothrombosis associated with plaque rupture. Arterioscler Thromb Vasc Biol 2005;25: $240-45$

13. Adams HP Jr, Bendixen BH, Kappelle LJ, et al; TOAST Investigators. Classification of subtype of acute ischemic stroke: definitions for use in a multicenter clinical trial. Stroke 1993;24:35-41

14. Yoon W, Jung MY, Jung SH, et al. Subarachnoid hemorrhage in a multimodal approach heavily weighted toward mechanical thrombectomy with Solitaire stent in acute stroke. Stroke 2013;44:414-19

15. Zaidat OO, Yoo AJ, Khatri P, et al; Cerebral Angiographic Revascularization Grading (CARG) Collaborators, STIR Revascularization working group, STIR Thrombolysis in Cerebral Infarction (TICI) Task Force. Recommendations on angiographic revascularization grading standards for acute ischemic stroke: a consensus statement. Stroke 2013;44:2650-63

16. Rovira A, Orellana P, Alvarez-Sabin J, et al. Hyperacute ischemic stroke: middle cerebral artery susceptibility sign at echo-planar gradient-echo MR imaging. Radiology 2004;232:466-73

17. Cho KH, Kim JS, Kwon SU, et al. Significance of susceptibility vessel sign on $\mathrm{T} 2^{*}$-weighted gradient echo imaging for identification of stroke subtypes. Stroke 2005;36:2379-83

18. Sato $\mathrm{Y}$, Ishibashi-Ueda $\mathrm{H}$, Iwakiri $\mathrm{T}$, et al. Thrombus components in cardioembolic and atherothrombotic strokes. Thromb Res 2012; 130:278-80

19. Sato Y, Hatakeyama K, Yamashita A, et al. Proportion of fibrin and platelets differs in thrombi on ruptured and eroded coronary atherosclerotic plaques in humans. Heart 2005;91:526-30

20. Patel MR, Edelman RR, Warach S. Detection of hyperacute primary intraparenchymal hemorrhage by magnetic resonance imaging. Stroke 1996;27:2321-24 